\title{
Simplified modelling and analysis of a rotating Euler-Bernoulli beam with a single cracked edge.
}

\author{
Ahmed Yashar ${ }^{\mathrm{a}, *}$, Neil Ferguson ${ }^{\mathrm{a}}$, Maryam Ghandchi-Tehrani ${ }^{\mathrm{a}}$ \\ ${ }^{a}$ Institute of Sound and Vibration Research, University of Southampton, \\ Southampton SO17 1BJ, UK
}

\begin{abstract}
The natural frequencies and mode shapes of the flapwise and chordwise vibrations of a rotating cracked Euler-Bernoulli beam are investigated using a simplified method. This approach is based on obtaining the lateral deflection of the cracked rotating beam by subtracting the potential energy of a rotating massless spring, which represents the crack, from the total potential energy of the intact rotating beam. With this new method, it is assumed that the admissible function which satisfies the geometric boundary conditions of an intact beam is valid even in the presence of a crack. Furthermore, the centrifugal stiffness due to rotation is considered as an additional stiffness, which is obtained from the rotational speed and the geometry of the beam. Finally, the Rayleigh-Ritz method is utilised to solve the eigenvalue problem. The validity of the results is confirmed at different rotational speeds, crack depth and location by comparison with solid and beam finite element model simulations. Furthermore, the mode shapes are compared with those obtained from finite element models using a Modal Assurance Criterion (MAC).
\end{abstract}

Keywords: Rotating cracked beam, Rayleigh-Ritz method

\footnotetext{
*Corresponding author

Email address: amiy1g14@soton.ac.uk.com (Ahmed Yashar)
} 


\section{Nomenclature}

a Crack depth

$b \quad$ Beam width

$d \quad$ The displacement vector for flapwise motion

$f \quad$ The transverse lateral distributed force

$h \quad$ Beam height

$n \quad$ The number of admissible functions

$r \quad$ Hub radius

$t \quad$ Time

$w \quad$ The transverse displacement

$x \quad$ Position along the beam in $X$ diration

$\bar{d} \quad$ An arbitrary vector with the same dimensions of $d$

A Cross sectional area

C Flexibility constant

E Young's modulus of elasticity

$F \quad$ A correction factor

I The second moment of area

$K E \quad$ Kinetic energy

$K_{c} \quad$ The stress intensity factor (SIF)

L Beam length

$M_{b} \quad$ Bending moment

$N \quad$ The admissible functions

O Centre of rotation 
$P \quad$ The longitudinal (axial) force

$P E \quad$ Strain energy

$T \quad$ The tensile force

$X, Y, Z$ Coordinate system fixed on the beam

$Y_{w} \quad$ A shape function of static deformation of a cantelever beam subjected to lateral load on the free-end

G The gyroscopic matrix

K The stiffness matrix

M The mass matrix

S The rotational motion-induced stiffness matrices

$\alpha \quad$ The Ritz coefficients

$\mu \quad$ The longitudinal deflection

$\omega \quad$ Frequency

$\phi \quad$ The rotational deflection

$\rho \quad$ Mass density

$\sigma \quad$ Stress

$\Theta \quad$ Slope

$\varpi \quad$ An arbitrary weight function

$\xi \quad$ Crack depth ratio

$\Omega \quad$ Rotational speed (rpm)

$\Pi \quad$ The elastic energy available per unit increase in the crack surface area

$w, v, s$ Subscript representing flapwise, chordwise and stretch 


\section{Introduction}

Vibration analysis of rotating beams is an important requirement in power generation or aviation, due to the substantial role in various applications such as gas turbine blades, wind turbines and helicopter propellers. Fractures are the most common faults in these structures, which contribute to increased vibration, ultimately causing complete failure of the structures. Research has been carried out concerning the vibrational behaviour of a cracked rotating blade, including crack identification and detection methods so that subsequent damage could be prevented.

Numerous studies have investigated the effect of the rotational speed on the natural vibration of cantilever beams. These studies concluded that the natural frequencies of flexural vibration tended to increase above those for the non-rotating beams, because of the centrifugal force and the subsequent stiffening effects [1]. The free vibration analysis of rotating beams have been widely studied using different numerical approximation methods, such as, the Rayleigh or Rayleigh-Ritz methods [1, 2, 3, 4, 5], the finite element method $[6,7,8,9,10]$, the differential transform method [11] and Galerkin's method [12]. Moreover, AL-Said et al [13, 14] analysed the free vibration of a Timoshenko beam modelled by a massless torsional spring, to simulate the flapwise vibration of a thick rotating beam. The admissible functions assumed were for the non rotating cracked beam either side of the crack. The various methods give clear evidence of the efforts made in finding a simple model that reproduces the rotational effect on rotating beams. However, the analysis and publications covering rotating cracked beams are relatively scarce in the literature $[15,16]$.

Furthermore, there is significant amount of research that has investigated crack modelling and its effects on non-rotating beams $[17,18,19,20,21$, 22, 23, 24]. Fernandez-Saez et al. used the Rayleigh method to estimate the fundamental frequency for bending vibration of a non-rotating cracked beam. A cracked rectangular and circular cross section beam were modelled using the finite element method [17]. In addition to a single crack, a multi cracked beam was modelled by Mazanoglu et al [18, 19] using the RayleighRitz method for a cracked non-uniform beam.

The objective of this paper is to develop a simplified method to model a cracked rotating cantilever beam. This approach is achieved by selecting the simplest admissible function of an un-cracked and non-rotating uniform cantilever beam. Then, the effect of beam rotation and flexibility of the cracked 
edge will be considered in obtaining the potential energy of the structure.

Initially, the un-cracked rotating beam is modelled using a simple continuous beam. The equilibrium of forces and application of Hamilton's principle is then used to find the governing differential equations [8]. Subsequently, a crack is introduced using fracture mechanics as presented by Dimarogonas [25]. Then, a Rayleigh Ritz method is used to obtain the numerical results for a rotating cracked beam. Finally, a parametric study is carried out for a cracked rotating beam using parameters such as the rotational speed, the crack depth and its location. In addition, the mode shapes are obtained and compared with the numerical results from finite element models using the Modal Assurance Criterion (MAC). From the modes identified, the veering in the dispersion curve for the natural frequencies versus rotational speed can be both inspected and interpreted.

\section{Theoretical modelling of a rotating cracked beam}

A rotating beam is considered as a homogeneous, Euler-Bernoulli uniform and isotropic cantilever beam of length $L$, with cross sectional area $A$, Young's modulus of elasticity $E$ and mass density $\rho$. It is attached to a rigid hub with radius $r$ about an axis through point $O$ as shown in Figure 1 . The cantilever beam and the rigid hub are rotating about the vertical axis $Z$ with angular velocity $\Omega$. The cantilever beam's length is along the $X$ axis. The flapwise vibration occurs in the $X Z$ plane, perpendicular to the plane of rotation. This coordinate system $(X, Y, Z)$ rotates with the hub.

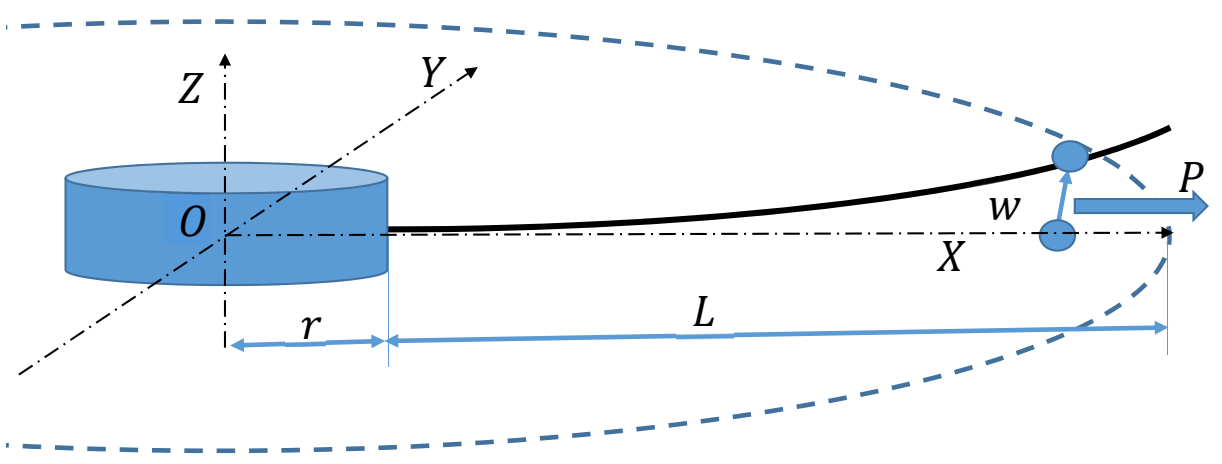

Figure 1: Configuration of rotating beam with length $L$ in the $X Y Z$ coordinate system subject to an axial centrifugal force $P$ and transverse deflection $w$ 
According to many researchers $[12,26,16]$, the equation of motion for the lateral vibration of a uniform thin homogeneous beam subjected to an axial force can be written as;

$$
\rho A \frac{\partial^{2} w}{\partial t^{2}}+\frac{\partial^{2}}{\partial x^{2}}\left(E I \frac{\partial^{2} w}{\partial x^{2}}\right)-\frac{\partial}{\partial x}\left(P \frac{\partial w}{\partial x}\right)=f(x, t)
$$

where $I, w, P$ and $f$ represent the second moment of area, transverse displacement, longitudinal (axial) force and transverse lateral distributed force respectively. For a rotating beam, the axial force $P$ is equal to the centrifugal force as shown in Figure 1 and can be obtained as a function of the position $x$ along the beam from the hub outer radius, where,

$$
P(x)=\int_{x}^{L} \rho A \Omega^{2}(x+r) d x=\rho A \Omega^{2}\left\{r(L-x)+\frac{1}{2}\left(L^{2}-x^{2}\right)\right\}
$$

The equation of motion for the transverse vibration of the rotating beam can then be obtained by substituting equation (2) into equation (1);

$$
\rho A \frac{\partial^{2} w}{\partial t^{2}}+\frac{\partial^{2}}{\partial x^{2}}\left(E I \frac{\partial^{2} w}{\partial x^{2}}\right)-\rho A \Omega^{2} \frac{\partial}{\partial x}\left\{\left[r(L-x)+\frac{1}{2}\left(L^{2}-x^{2}\right)\right] \frac{\partial w}{\partial x}\right\}=0
$$

For the free vibration of the rotating beam when $f(x, t)=0$, a harmonic solution for the displacement with the frequency $\omega$ and amplitude $W$ of the separable following form is assumed.

$$
w(x, t)=W(x) \cos (\omega t)
$$

Substituting the solution, 4 , into 3 yields,

$$
-\rho A W \omega^{2}+\frac{d^{2}}{d x^{2}}\left(E I \frac{d^{2} W}{d x^{2}}\right)-\rho A \Omega^{2} \frac{d}{d x}\left\{\left[r(L-x)+\frac{1}{2}\left(L^{2}-x^{2}\right)\right] \frac{d W}{d x}\right\}=0
$$

The boundary conditions are assumed to be as follows for a cantilever beam;

$$
\begin{aligned}
W(x)=\frac{d W(x)}{d x}=0 & , \quad x=0 \\
E I \frac{d^{2} W(x)}{d x^{2}}=E I \frac{d^{3} W(x)}{d x^{3}}=0 & , \quad x=L
\end{aligned}
$$

The exact solution of equation (5) is difficult to obtain analytically due to the inclusion of the centrifugal force and the term $\frac{d}{d x}\left(x^{2} \frac{d W}{d x}\right)$. Nevertheless, 
an approximate method using a numerical method such as the finite element method or Rayleigh-Ritz method can be used to solve this equation. The strong form for the solution is not sought, instead an equivalent weak form is expressible by multiplication of arbitrary weight function $\varpi$ and integration over the domain, which is the length of the beam $L$.

$$
\begin{array}{r}
\int_{0}^{L} \varpi\left(\rho A \frac{\partial^{2} w}{\partial t^{2}}+\frac{\partial^{2}}{\partial x^{2}}\left(E I \frac{\partial^{2} w}{\partial x^{2}}\right)\right. \\
\left.-\rho A \Omega^{2} \frac{\partial}{\partial x}\left\{\left[r(L-x)+\frac{1}{2}\left(L^{2}-x^{2}\right)\right] \frac{\partial w}{\partial x}\right\}-f\right) d x=0
\end{array}
$$

The weighting and displacements functions are now approximated by the shape functions as

$$
\varpi=(\bar{d})^{T} N_{w}^{T} ; w=N_{w} d
$$

where $d$ and $\bar{d}$ are the displacement vector for flapwise displacements and an arbitrary vector with the same dimensions. $N$ represents the admissible functions and the subscript letter $w$ refers to the the flapwise direction. The admissible function $N_{w}$ is assumed as a vector summation of $n$ static deformation shapes of a non-rotating uncracked cantilever beam that is subjected to a concentrated load on the free end.

$$
\begin{aligned}
N_{w}(x) & =\sum_{i=1}^{n} \alpha_{i} Y_{w i}(x) \\
Y_{w n}(x) & =\left(\frac{x}{L}\right)^{(n-1)}\left(\frac{-L x^{2}}{2}+\frac{x^{3}}{6}\right)
\end{aligned}
$$

where $\alpha_{i}$ represents the constant Ritz coefficients satisfying the boundary conditions and $n$ is the number of shape functions used.

Introducing the approximate solutions given by equation 8 and substituting into the weak equations given by equation 7 , the equations of motion can also be written in a matrix form, yielding the discretised equations for the flapwise motion as

$$
\left(\overline{d_{w}}\right)^{T}\left[\mathbf{M}_{w} \ddot{d}_{w}+\left(\mathbf{K}_{w}+\Omega^{2} \mathbf{S}_{w}\right) d_{w}\right]=\left(\overline{d_{w}}\right)^{T} \mathbf{f}_{w}
$$

where $\mathbf{M}_{w}, \mathbf{K}_{w}$ and $\mathbf{S}_{w}$ are the mass, stiffness and motion-induced stiffness matrices for the flapwise motion. $\mathbf{f}_{w}$ is the load vector for the flapwise motion. 
The subscript letters $w$ denote the flapwise direction and the matrix terms are

$$
\begin{aligned}
\mathbf{M}_{w} & =\rho A \int_{0}^{L} N_{w} N_{w}^{T} d x, \\
\mathbf{K}_{w} & =E I_{y} \int_{0}^{L} \frac{d^{2} N_{w}}{d x^{2}} \frac{d^{2} N_{w}^{T}}{d x^{2}} d x, \\
\mathbf{S}_{w} & =\rho A \int_{0}^{L}\left[r(L-x)+\frac{1}{2}\left(L^{2}-x^{2}\right)\right] \frac{d N_{w}}{d x} \frac{d N_{w}^{T}}{d x} d x, \\
\mathbf{f}_{w} & =p_{w} \int_{0}^{L} N_{w} d x,
\end{aligned}
$$

According to Chung and Yoo [8], the chordwise vibration is coupled with longitudinal or extensional vibration due to a gyroscopic effect and they are both uncoupled with the flapwise vibration. Furthermore, the chordwise vibration can be written as [8],

$$
\left(d_{s v}\right)^{T}\left\{\mathbf{M}_{s v} \ddot{d}_{s v}+2 \Omega \mathbf{G}_{s v} \dot{d}_{s v}+\left[\mathbf{K}_{s v}+\Omega^{2}\left(\mathbf{S}_{s v}-\mathbf{M}_{s v}\right)+\dot{\Omega} \mathbf{G}_{s v}\right] d_{s v}\right\}=\left(d_{s v}\right)^{T} \mathbf{f}_{s v}
$$

where subscript letters $s$ and $v$ represent stretch and chordwise directions respectively. $\mathbf{M}_{s v}, \mathbf{K}_{s v}, \mathbf{G}_{s v}, \mathbf{S}_{s v}$ and $\mathbf{f}_{s v}$ are the corresponding mass, stiffness, gyroscopic, rotational stiffness and external force matrices.

$$
\begin{aligned}
\mathbf{M}_{s v} & =\rho A \int_{0}^{L}\left(N_{s} N_{s}^{T}+N_{v} N_{v}^{T}\right) d x, \\
\mathbf{G}_{s v} & =\rho A \int_{0}^{L}\left(N_{v} N_{s}^{T}+N_{s} N_{v}^{T}\right) d x \\
\mathbf{K}_{s v} & =\int_{0}^{L}\left(E A \frac{d N_{s}}{d x} \frac{d N_{s}^{T}}{d x}+E I_{z} \frac{d^{2} N_{v}}{d x^{2}} \frac{d^{2} N_{v}^{T}}{d x^{2}}\right) d x, \\
\mathbf{S}_{s v} & =\rho A \int_{0}^{L}\left[r(L-x)+\frac{1}{2}\left(L^{2}-x^{2}\right)\right] \frac{d N_{v}}{d x} \frac{d N_{v}^{T}}{d x} d x, \\
\mathbf{f}_{s v} & =\int_{0}^{L}\left\{\rho A \Omega^{2}(r+x) N_{s}+\left[p_{v}-\rho A \dot{\Omega}(r+x)\right] N_{v}\right\} d x,
\end{aligned}
$$

where the static deflection function for the chordwise bending and longitudinal vibration can be expressed as 


$$
\begin{aligned}
& Y_{v n}(x)=\left(\frac{x}{L}\right)^{n-1}\left(\frac{-L x^{2}}{2}+\frac{x^{3}}{6}\right) \\
& Y_{s n}(x)=\left(\frac{x}{L}\right)^{n-1}\left(\frac{x}{L}\right)
\end{aligned}
$$

Regarding fracture mechanics theory, an open crack in a structure can be considered as a source of additional local flexibility because of the increase in the strain energy in the area surrounding the crack tip. The idea of substituting massless springs instead of a crack is to create the relation between the strain energy and the applied loads as shown in Figure (2).

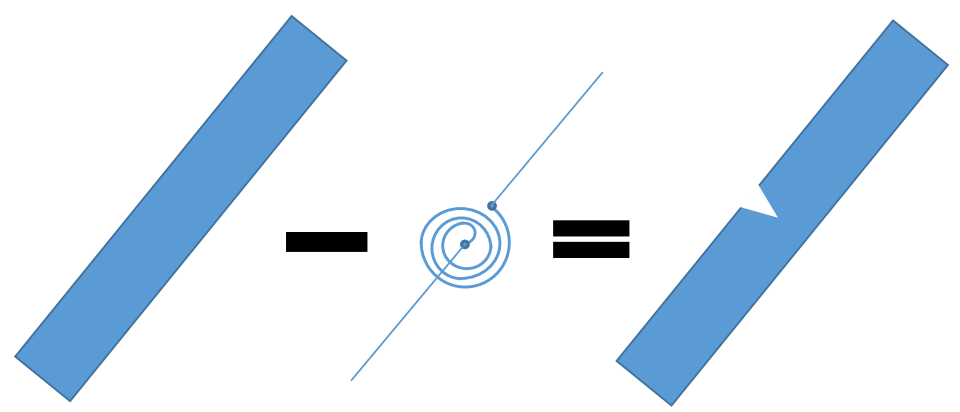

Figure 2: Modelling the crack using a massless torsional spring. The potential energy of the rotational massless spring is subtracted from the total potential energy of the intact beam

The typical method of modelling a cracked beam is based on dividing the beam into two segments and building a relationship between two sides using the compatibility conditions. The vertical displacement, bending moment and shear force are equal through the cracked edge. Nevertheless, the presence of the crack introduces a discontinuity or change in the slope of the beam, which is proportional to the bending moment that is transmitted through the cracked section. For the axial vibration, it is assumed that the tensile force is the same through the cracked segments. However, the axial displacement is proportional to the transmitted force through the crack, and it is proportional to the tensile force. The bending and axial discontinuity can be expressed as presented in [21] and [22].

$$
\begin{aligned}
\Delta \Theta & =C_{v, w} M_{b} \\
\Delta s & =C_{s} T
\end{aligned}
$$


where $\Delta \Theta, C, M_{b}$ represent slope discontinuity, axial flexibility constant and the bending moment transmitted through the crack section. $\Delta s$ and $T$ represent the discontinuity of axial displacement and tensile force respectively. The flexibility constant is dependent on the geometrical dimension of the cross section and can be derived from the stress intensity factor $K_{c}$ (SIF).

$$
K_{c}=\sigma \sqrt{\pi a} F\left(\frac{a}{h}\right)
$$

here $\sigma, a, h$ and $F$ are the stress according to the bending moment or tensile force, crack depth, beam height and correction factor respectively. $F$ depends on the crack's mode and force configurations. The stress $\sigma$ and the correction factor $F$ for the bending moment and opening mode can be obtained from $[17,27]$

$$
\begin{aligned}
\sigma_{M_{b}} & =\frac{6 M_{b}}{b h^{2}} \\
F_{I M_{b}} & =\sqrt{\frac{2}{\pi \xi} \tan \frac{\pi \xi}{2}} \frac{0.923+0.199\left[1-\sin \left(\frac{\pi \xi}{2}\right)\right]^{4}}{\sqrt{\cos \left(\frac{\pi \xi}{2}\right)}}
\end{aligned}
$$

where $b$ represents the beam width and the crack depth to thickness ratio $\xi=(a / h)$, while for the tensile and opening mode, are

$$
\begin{aligned}
\sigma_{T} & =\frac{T}{b h} \\
F_{I T} & =\sqrt{\frac{2}{\pi \xi} \tan \frac{\pi \xi}{2}} \frac{0.752+2.02 \xi+0.37\left[1-\sin \left(\frac{\pi \xi}{2}\right)\right]^{3}}{\sqrt{\cos \left(\frac{\pi \xi}{2}\right)}}
\end{aligned}
$$

These error functions are reported in [28] are accurate better than $0.5 \%$ for any crack depth ratio $\xi$. The total strain energy of the elastic deformation of the crack is given by,

$$
P E_{\text {total }}=\int_{A_{c}} \Pi d A_{c}
$$

where $\Pi$ is the elastic energy available per unit increase in the crack surface area.

$$
\Pi=\frac{K_{c}^{2}}{E}
$$


Substituting equations 26-32 into equation 31, yields

$$
\begin{aligned}
P E_{M_{b}} & =\left(\frac{3 \pi M^{2} h}{E I}\right) \int_{0}^{a}\left(\frac{a}{h^{2}}\right) F_{I M_{b}}^{2} d a \\
P E_{T} & =\frac{\pi P^{2} h}{E A} \int_{0}^{a}\left(\frac{a}{h^{2}}\right) F_{I T}^{2} d a
\end{aligned}
$$

where $P E_{M_{b}}$ is the strain energy due to the bending and $P E_{T}$ is the tensile strain energy due to tension. Utilizing Castigliano's theorem [23], the rotation $\phi$ and stretch $\mu$ corresponding to the applied moment and longitudinal force can be expressed as,

$$
\begin{gathered}
\phi=\frac{\partial P E_{M_{b}}}{\partial M_{b}} \\
\mu=\frac{\partial P E_{T}}{\partial T}
\end{gathered}
$$

Finally, the additional flexibility in flapwise $C_{w}$, chordwise $C_{v}$ and stretch $C_{s}$ directions due to the presence of the crack can be expressed as

$$
\begin{aligned}
C_{v}=C_{w} & =\frac{\phi}{M} \\
C_{s} & =\frac{\mu}{T}
\end{aligned}
$$

\section{Application of the Rayleigh-Ritz method}

According to this approach, an admissible function of the non-rotating intact beam satisfies the boundary conditions and it is used directly without any modification. The potential energy of the system is modified to include the effect of the extra flexibility due to the existence of the crack.

The potential energy of the non rotating intact beam can be expressed as,

$$
\begin{aligned}
P E_{M_{b}-\text { intact-nonrotate }} & =\frac{E I}{2} \int_{0}^{L} \frac{d^{2} N_{v, w}}{d x^{2}} \frac{d^{2} N_{v, w}^{T}}{d x^{2}} d x \\
P E_{T-\text { intact-nonrotate }} & =\frac{E A}{2} \int_{0}^{L} N_{s} N_{s}^{T} d x
\end{aligned}
$$


The additional potential energy due to the centrifugal effect on the intact beam can be written as,

$$
P E_{\text {centrifugal }}=\frac{1}{2} \Omega^{2} \rho A \int_{0}^{L}\left(r(L-x)+\frac{1}{2}\left(L^{2}-x^{2}\right) \frac{d N_{v, w}}{d x} \frac{d N_{v, w}^{T}}{d x} d x\right.
$$

Finally, the presence of additional flexibility due to the crack can be represented by a massless rotational spring and the corresponding potential energy in the spring has two components, which can be expressed as [24],

$$
\begin{aligned}
P E_{M_{b}-\text { crack }} & =\frac{1}{2}(\Delta \Theta) M_{b}=\frac{1}{2} E I C_{v, w}\left(\frac{d^{2} N_{v, w}}{d x^{2}} \frac{d^{2} N_{v, w}^{T}}{d x^{2}}\right) \\
P E_{T-\text { crack }} & =\frac{1}{2}(\Delta s) T=\frac{1}{2} E A C_{s}\left(N_{s} N_{s}^{T}\right)
\end{aligned}
$$

The kinetic energy of the system $K E$ is equal to the kinetic energy of the beam itself, i.e.

$$
\begin{aligned}
K E_{\text {flapwise }} & =\frac{1}{2} \rho A \int_{0}^{L} N_{w} N_{w}^{T} d x \\
K E_{\text {chordwise }} & =\frac{1}{2} \rho A \int_{0}^{L}\left(N_{s} N_{s}^{T}+N_{v} N_{v}^{T}\right) d x
\end{aligned}
$$

The Rayleigh-Ritz method can now be employed after determining the kinetic and potential energies of the system.

When applying the mode shapes of the uncracked beam as the admissible functions for the Rayleigh-Ritz approximation, the maximum strain energy needs to be altered to account for the reduction of the energy as a result of the presence of the crack. This energy reduction is proportional to the amount of additional flexibility due to the rotational spring. As a result, the total potential energy of the cracked rotating beam is equal to the summation of the potential energy of the uncracked beam and the additional potential energy due to the centrifugal force with subtraction of the potential energy of the massless rotating spring representing the crack.

$$
P E_{\text {total }}=P E_{\text {intact-nonrotate }}+P E_{\text {centrifugal }}-P E_{\text {crack }}
$$

Substituting equations 39-40, 41 and 42-43 into equation 46 provides the total strain energy of the system for the flapwise vibration and the equations 44-45 gives the kinetic energy. These two energies were used to calculate the flapwise and chordwise natural frequency of the rotating cracked cantilever beam. 


\section{Results and discussions}

The numerical results for the newly developed simple model are compared with a model built using one dimensional beam finite elements. Each element has two nodes and each node has two degrees of freedom in the case of the flapwise model, which are lateral displacement and slope. The elements are chosen to have three degrees of freedom in the case of the chordwise model, which are lateral displacement, slope and longitudinal displacement [29]. The results are also compared with a solid 3D element model assembled and solved using ANSYS. The solid model was built using a mesh of hexahedral beam elements and with a fine sized mesh around the crack tip as shown in Figure 3. The frequency ratio for the natural frequencies of the rotating cracked beam for different fixed rotational speeds against the fundamental frequency of the uncracked beam are shown in Figure 4. The natural frequencies all increase as the rotational speed increases. The estimated values using the Rayleigh-Ritz method show good agreement with the values from the one dimensional $\mathrm{FE}$ model with a maximum error less than $0.5 \%$ in the third mode.

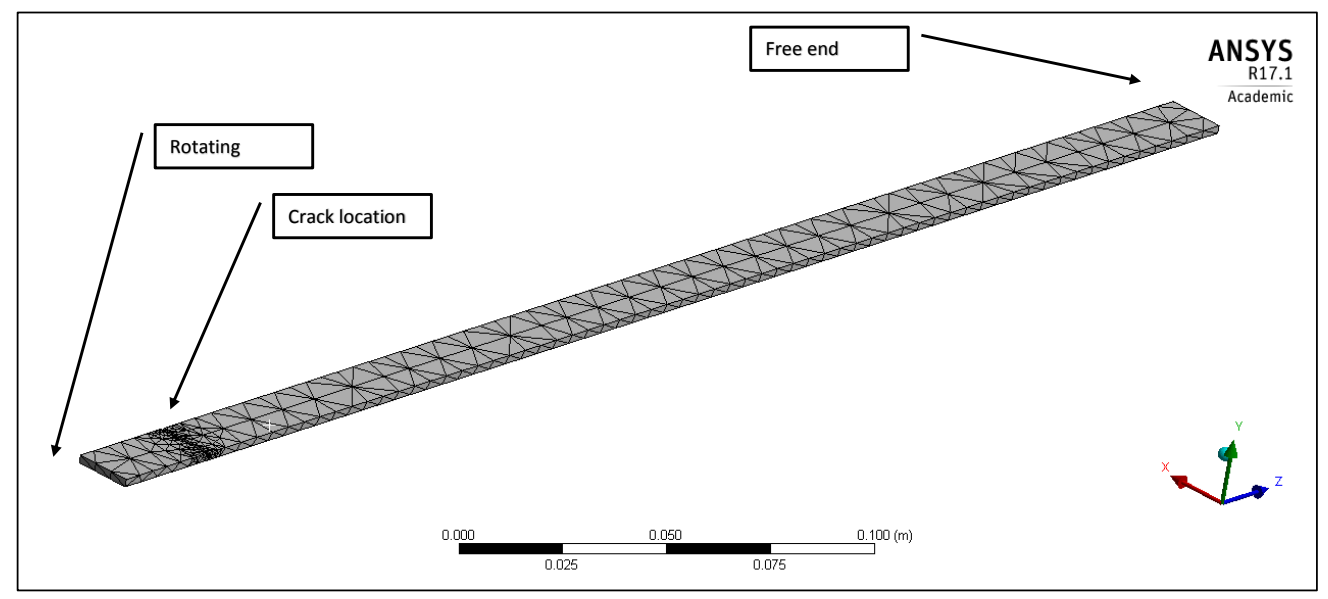

Figure 3: Rotating cracked cantilever beam model using 3D hexahedral FE elements in ANSYS.

Figure 5 shows the effect of the crack location on the natural frequencies of the rotating beam. The closer the crack is to the hub supporting the cantilever beam, the greater the reduction in the natural frequencies. Moreover, when the crack is located at a modal node, its effect becomes less evident, 

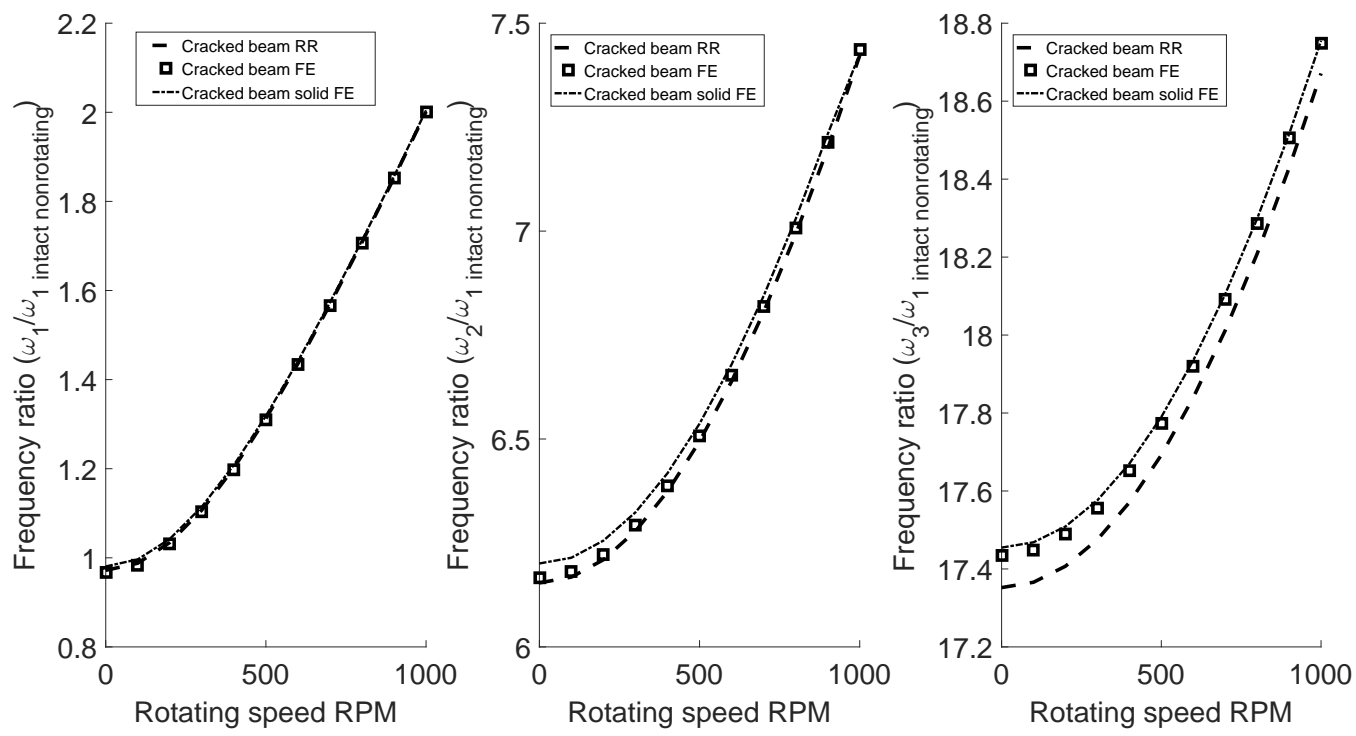

Figure 4: Lowest three flapwise natural frequencies of a rotating cracked beam versus the rotational speed. Crack location ratio is 0.075 of the beam length, crack depth ratio is 0.5 of the beam thickness. The maximum error is about $0.5 \%$ in the third mode.

as shown for the second and third modes in Figure 5. The maximum error occurs in the first mode when the crack is located at the root (fixed end) of the rotating beam.

In addition to the natural frequency comparison, the mode shapes of the cracked rotating blades were evaluated and compared with the corresponding modes using the finite element model. Figures 6 show the Modal Assurance Criterion (MAC) between the results of the present work and the one dimensional FE model. The diagonal white squares indicate the good agreement between the mode shapes using the two approaches. 

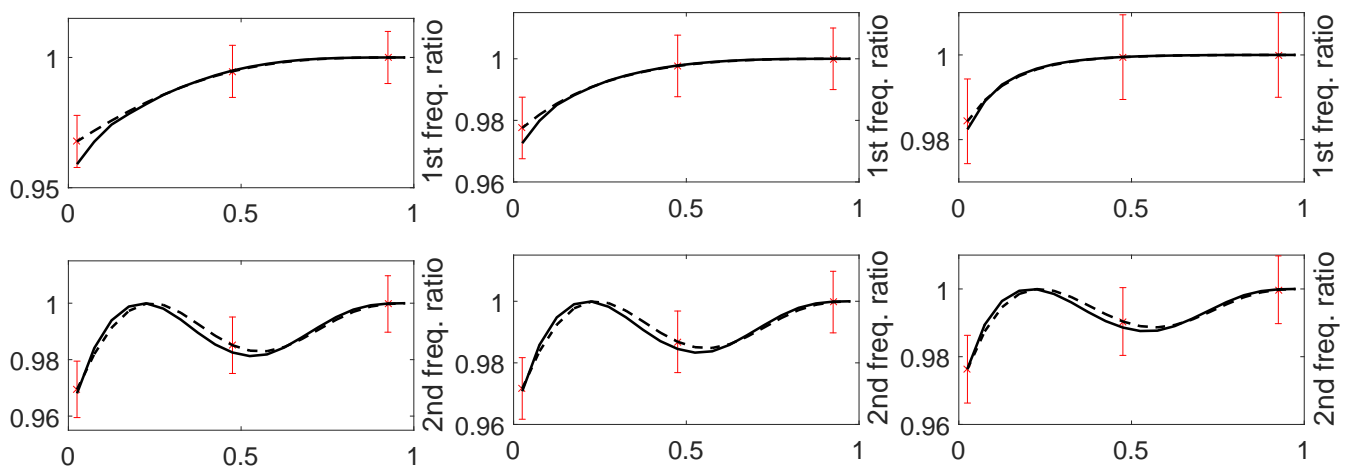

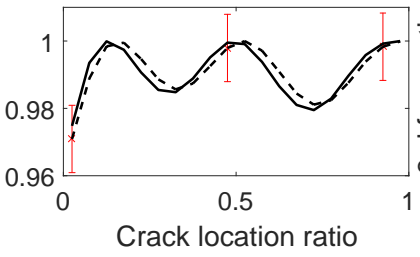

(a)

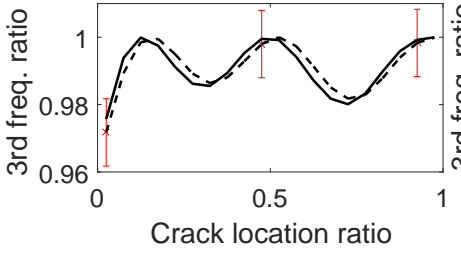

(b)

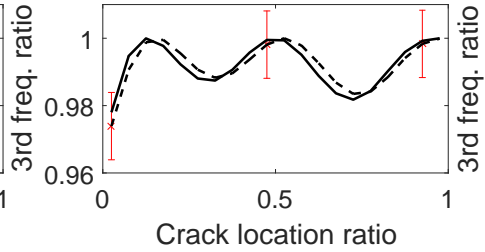

(c)

Figure 5: Frequency versus crack location as a ratio of crack position divided by beam length for the lowest three flapwise frequencies of a rotating cracked beam, for three different rotational speeds ((a) non-rotating, (b) for $500 \mathrm{rpm}$ and (c) for $1000 \mathrm{rpm}$ ) and crack depth ratio $\xi$ of 0.5 . Solid and dashed lines refer to the proposed method and FEM results respectively. The error bars are $1 \%$ difference.

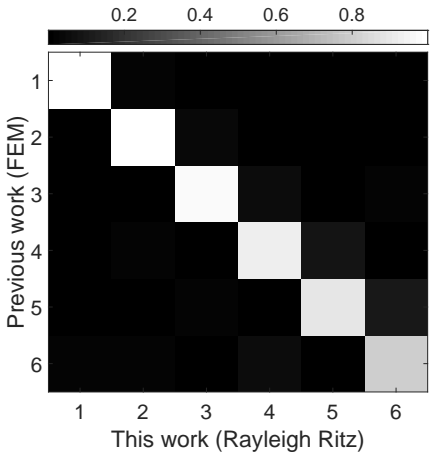

(a)

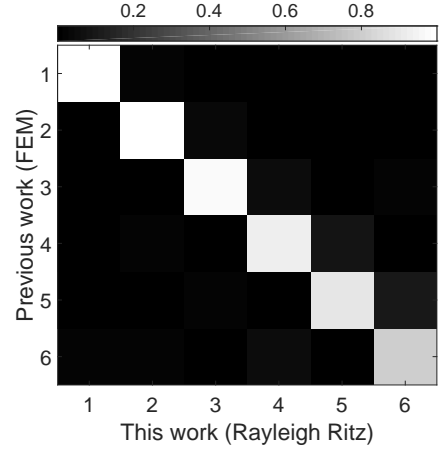

(b)

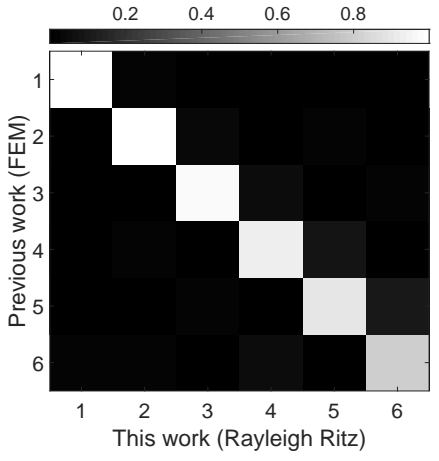

(c)

Figure 6: MAC diagrams for the lowest six modes of the flapwise vibration of the rotating cracked beam with the crack location ratio of 0.075 and crack depth ratio of 0.5 and rotational speeds (a) non-rotating, (b) $500 \mathrm{rpm}$ and (c) $1000 \mathrm{rpm}$. 
Regarding chordwise vibration, similar comparison was made for the lowest three natural frequencies with a model built using one-dimensional beam finite elements [29] and a solid finite element model using ANSYS. The frequency ratio between the natural frequency of the rotating cracked beam for different fixed rotational speeds against the fundamental frequency of the uncracked beam are shown in Figure 7. The estimated values using the Rayleigh-Ritz method show good agreement with the values obtained from one dimensional FE models having a maximum error of less than $0.77 \%$ in the third mode.

Furthermore, the crack location versus the natural frequency was compared in the chordwise vibration with the FE model and shows good agreement with a maximum error less than $1 \%$ for the at first natural frequency when the crack is close to the root of the cantilever beam. Likewise, the mode shapes of the chordwise vibration cracked rotating beams were evaluated and compared with the corresponding modes produced from the finite element model. Figure 9 shows the Modal Assurance Criterion (MAC) between the results of the proposed method and the FE model. The diagonal white squares indicate the good agreement for the mode shape matching between the two approaches.

Figure 10 shows the flapwise and chordwise natural frequency ratios versus rotational speed for the same cross sectional dimensions and identical crack location and depth. The chordwise vibration shows less sensitivity to the rotational speed than the flapwise vibration, due to the gyroscopic coupling effect in the chordwise vibration.

The coupling between bending and longitudinal displacements in the chordwise plane leads to a veering phenomena in the natural frequency versus rotational speed graph as can be seen in Figure 11. In the third lowest natural frequency, the bending chordwise mode natural frequency veers to become a stretch mode at the speed of $5900 \mathrm{rpm}$. The fourth is veering from bending to stretch at a rotational speed of $3800 \mathrm{rpm}$, then veering from stretch to bending again at a speed $5900 \mathrm{rpm}$. A similar phenomena appears in the fifth to seventh natural frequencies at the rotational speeds of 2800 , 2050 and $1450 \mathrm{rpm}$ respectively. 

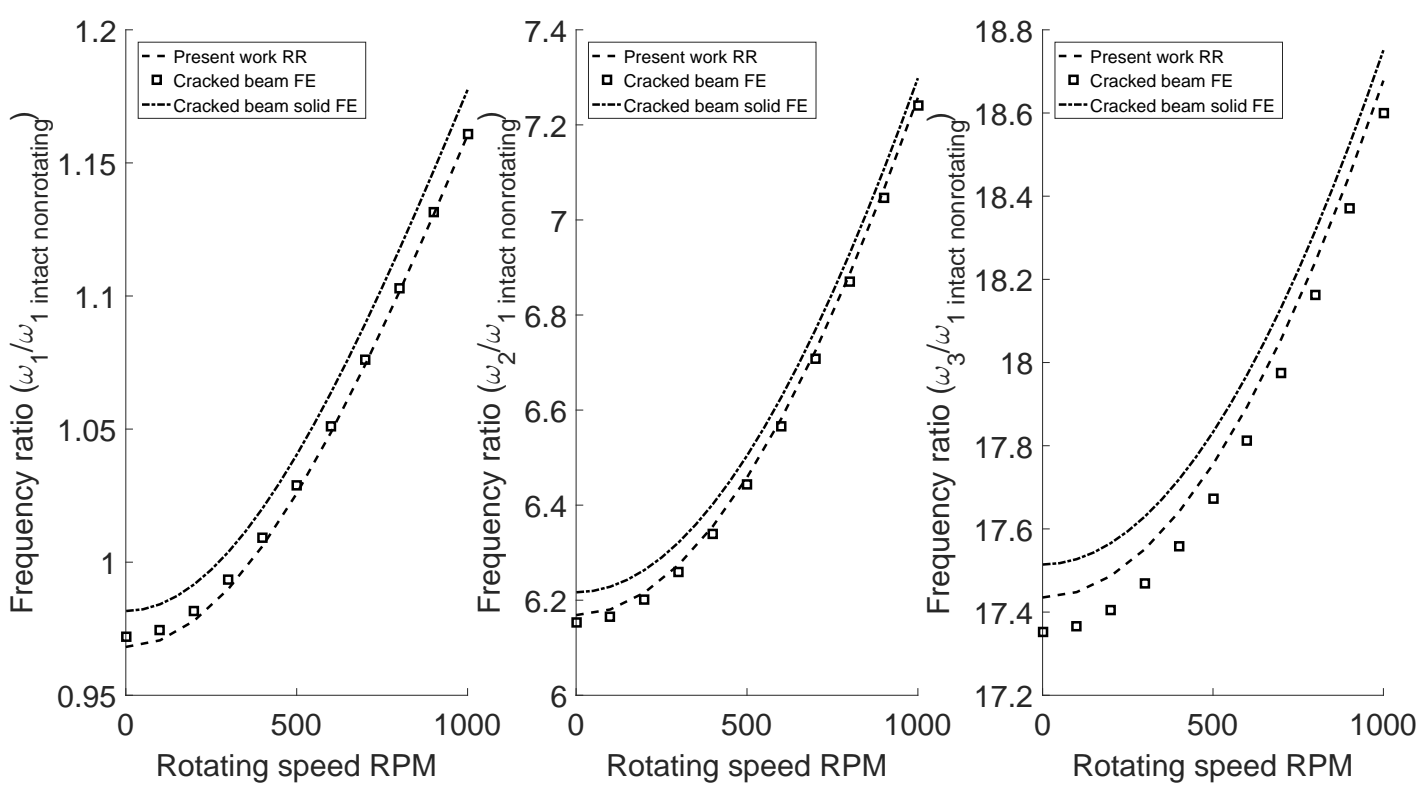

Figure 7: Lowest three chordwise natural frequencies of a rotating cracked beam versus the rotational speed. Crack location ratio is 0.075 of the beam length, crack depth ratio is 0.5 of the beam thickness. The maximum error is about $0.77 \%$ in the third mode. 

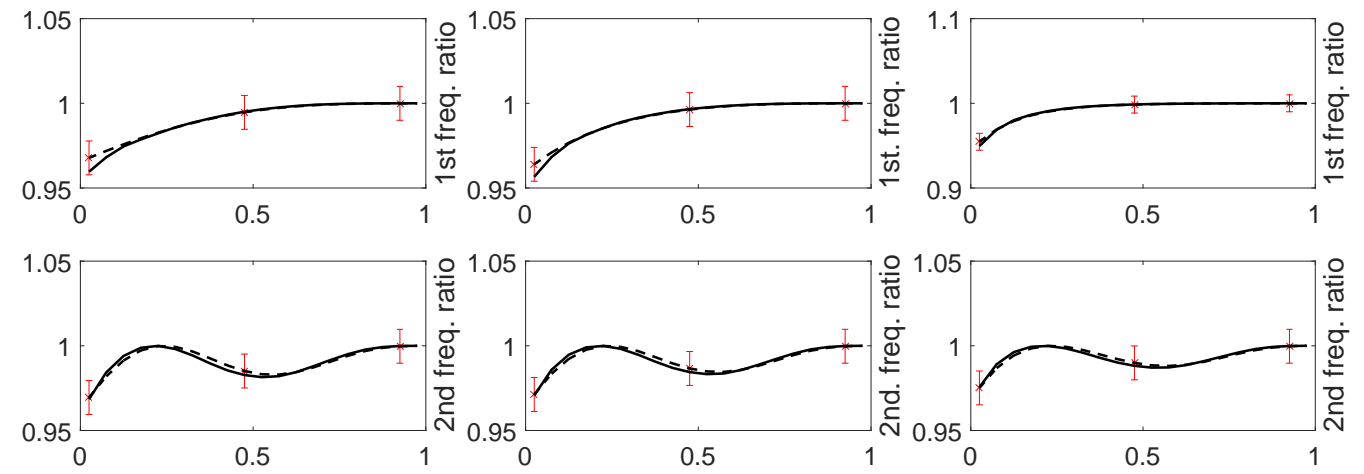

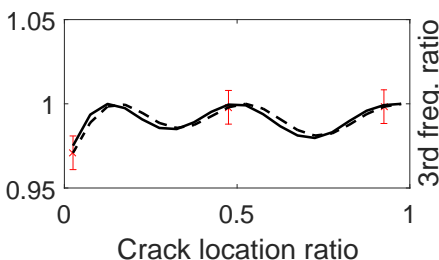

(a)

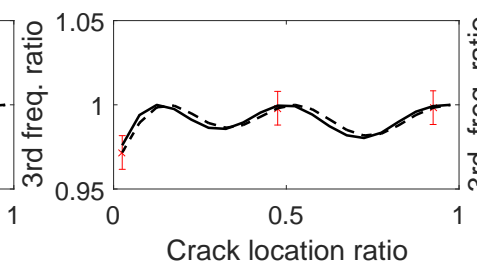

(b)

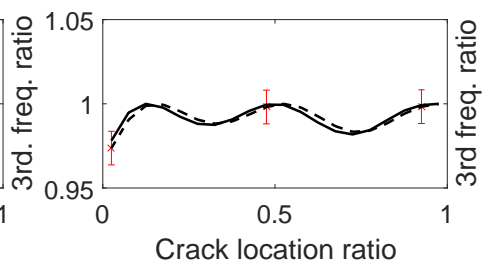

(c)

Figure 8: Frequency versus crack location for the lowest three chordwise natural frequencies of rotating cracked beam with three different rotational speeds ((a) non-rotating, (b) for $500 \mathrm{rpm}$ and (c) for $1000 \mathrm{rpm}$ ) and crack depth ratio $\xi$ of 0.5 . Solid and dashed lines refer to the proposed method and FEM respectively. The error bars are 1\% difference.

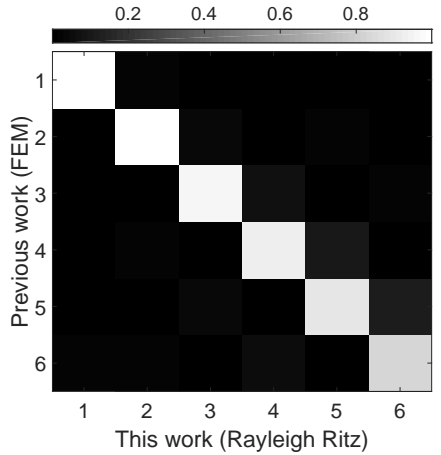

(a)

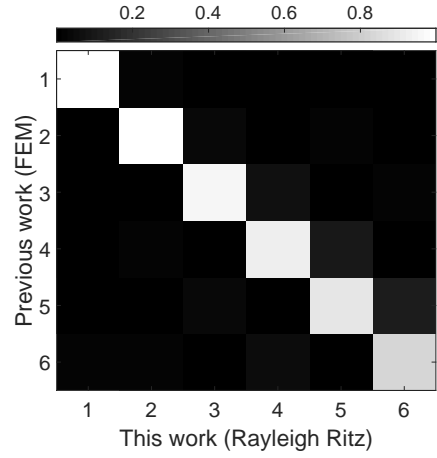

(b)

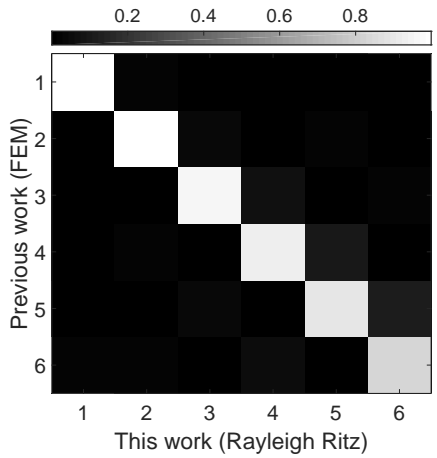

(c)

Figure 9: MAC diagrams for the lowest six modes of the chordwise vibration of the rotating cracked beam with the crack location ratio of 0.075 and crack ratio of 0.5 and rotational speeds (a) non-rotating, (b) $500 \mathrm{rpm}$ and (c) $1000 \mathrm{rpm}$. 


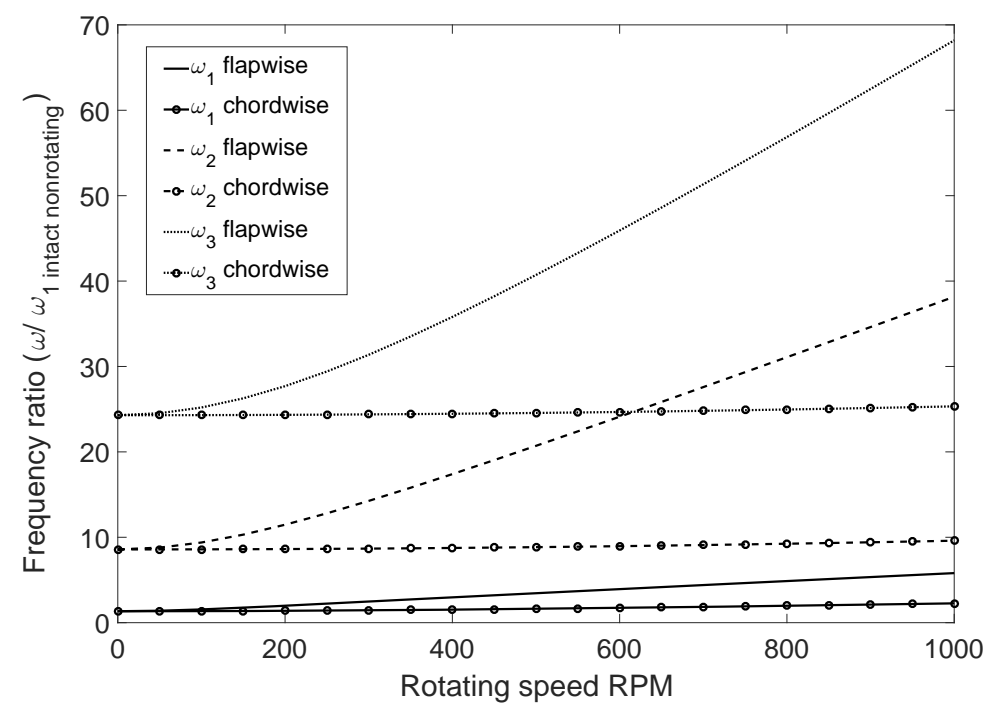

Figure 10: Lowest three flapwise and chordwise natural frequencies of a rotating cracked beam versus the rotational speed of the same cross sectional dimension. The crack location ratio is 0.075 and the crack depth ratio is 0.5 


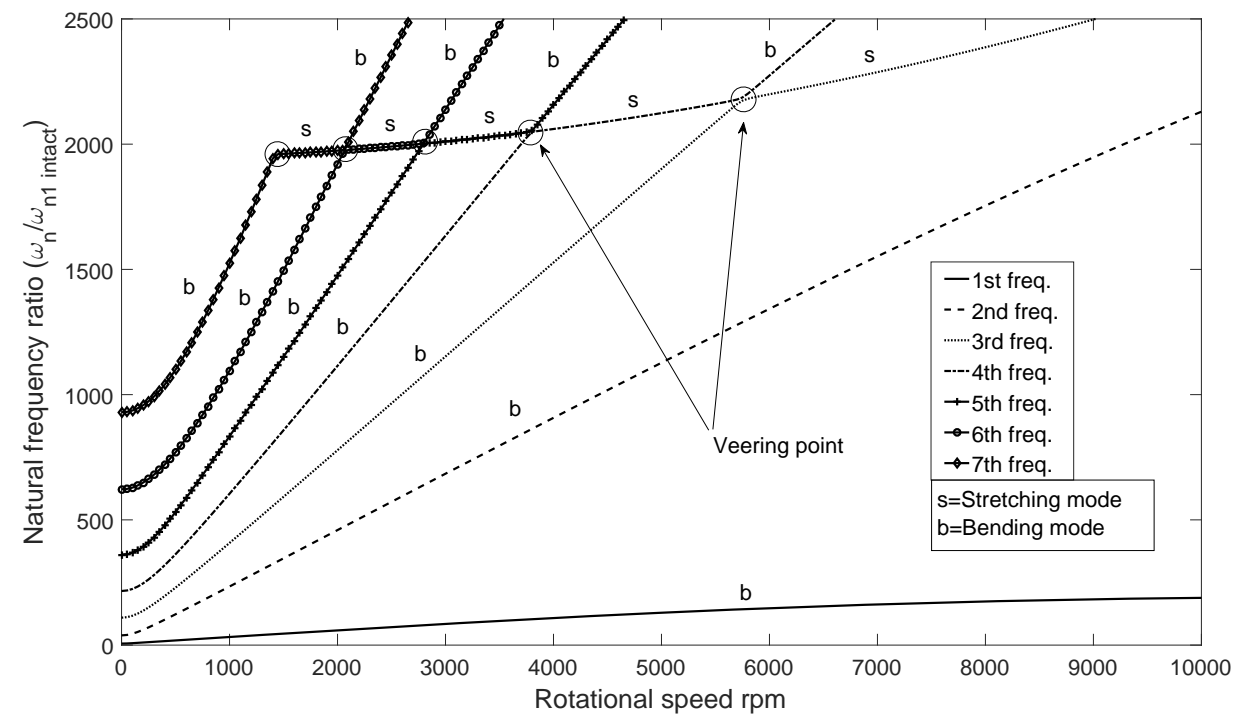

Figure 11: The veering of the bending and stretching natural frequencies at high rotational speed in the chordwise plane for the rotating cracked beam. The seven lowest natural frequencies are shown where s represents stretching and $\mathrm{b}$ represents bending modes. The crack location ratio is 0.075 of the beam length and the depth ratio is 0.5 of the beam thickness. 


\section{Conclusions}

A new simplified model for a rotating cracked beam was presented, based on a modified potential energy. This was used in a Rayleigh-Ritz approach for an Euler-Bernoulli beam model, rather than separating a cracked beam pre and post the crack which would require to separate additional polynomial shape functions. The approach herein was implemented and showed a good agreement, especially for the lowest three modes of the vibration, with FE numerical models using one-dimensional beam and solid elements. The maximum natural frequency error between the methods proposed and FE was less than $0.5 \%$ for the flapwise and less than $0.77 \%$ for the chordwise. Furthermore, the predicted vibration mode shapes matched well with the corresponding mode shapes evaluated using the FE model as shown with MAC plots. The bending vibration in the chordwise direction showed less sensitivity to the rotational speed than the bending vibration in the flapwise direction due to the gyroscopic coupling. Moreover, a veering phenomena clearly appeared in the higher natural frequencies for the chordwise vibration due to gyroscopic coupling at high rotational speeds.

Overall, this approach showed the possibility of selecting the shape functions for the Rayleigh-Ritz method, which satisfied only the beam end boundary conditions is sufficient for modelling a cracked beam. Other researchers $[13,14]$ used pre and post crack admissible functions for the Timoshenko beam case. Furthermore, the use of the Rayleigh-Ritz method significantly reduced the size of the numerical model when compared to alternatives such as the finite element models.

\section{Acknowledgements}

The lead author gratefully acknowledges the Ministry of Higher Education and Scientific Research in Iraq for providing a full PhD scholarship.

\section{References}

[1] N. Rubinstein, J. T. Stadter, Bounds to bending frequencies of a rotating beam, Journal of the Franklin Institute 294 (1972) 217-229.

[2] R. B. Bhat, Transverse vibrations of a rotating uniform cantilever beam with tip mass as predicted by using beam characteristic orthogonal polynomials in the Rayleigh-Ritz method, Journal of Sound and Vibration 105 (1986) 199-210. 
[3] H. Yoo, S. Shin, Vibration analysis of rotating cantilever beams, Journal of Sound and Vibration 212 (1998) 807-828.

[4] H. H. Yoo, J. E. Cho, J. Chung, Modal analysis and shape optimization of rotating cantilever beams, Journal of Sound and Vibration 290 (2006) $223-241$.

[5] K. Mazanoglu, S. Guler, Flap-wise and chord-wise vibrations of axially functionally graded tapered beams rotating around a hub, Mechanical Systems and Signal Processing 89 (2016) 97-107.

[6] S. Rao, R. Gupta, Finite element vibration analysis of rotating timoshenko beams, Journal of Sound and Vibration 242 (2001) 103-124.

[7] G.-P. Cai, J.-Z. Hong, S. X. Yang, Model study and active control of a rotating flexible cantilever beam, International Journal of Mechanical Sciences 46 (2004) 871-889.

[8] J. Chung, H. Yoo, Dynamic Analysis of a Rotating Cantilever Beam By Using the Finite Element Method, Journal of Sound and Vibration 249 (2002) 147-164.

[9] J. B. Yang, L. J. Jiang, D. C. Chen, Dynamic modelling and control of a rotating Euler-Bernoulli beam, Journal of Sound and Vibration 274 (2004) 863-875.

[10] J. R. Banerjee, H. Su, D. R. Jackson, Free vibration of rotating tapered beams using the dynamic stiffness method, Journal of Sound and Vibration 298 (2006) 1034-1054.

[11] O. Ozdemir, M. O. Kaya, Flapwise bending vibration analysis of a rotating tapered cantilever Bernoulli-Euler beam by differential transform method, Journal of Sound and Vibration 289 (2006) 413-420.

[12] H. Kim, H. Hee Yoo, J. Chung, Dynamic model for free vibration and response analysis of rotating beams, Journal of Sound and Vibration 332 (2013) 5917-5928.

[13] S. M. Al-Said, M. Naji, A. A. Al-Shukry, Flexural vibration of rotating cracked Timoshenko beam, Journal of Vibration and Control 12 (2006) $1271-1287$. 
[14] A. A. Masoud, S. Al-Said, A new algorithm for crack localization in a rotating Timoshenko beam, Journal of Vibration and Control 15 (2009) $1541-1561$.

[15] A. Banerjee, G. Pohit, Crack Investigation of rotating cantilever beam by fractal dimension analysis, Procedia Technology 14 (2014) 188-195.

[16] Y. Cheng, Z. Yu, X. Wu, Y. Yuan, Vibration analysis of a cracked rotating tapered beam using the p-version finite element method, Finite Elements in Analysis and Design 47 (2011) 825-834.

[17] D. Y. Zheng, N. J. Kessissoglou, Free vibration analysis of a cracked beam by finite element method, Journal of Sound and Vibration 273 (2004) 457-475.

[18] K. Mazanoglu, I. Yesilyurt, M. Sabuncu, Vibration analysis of multiplecracked non-uniform beams, Journal of Sound and Vibration 320 (2009) 977-989.

[19] K. Mazanoglu, M. Sabuncu, Flexural vibration of non-uniform beams having double-edge breathing cracks, Journal of Sound and Vibration 329 (2010) 4181-4191.

[20] A. Ariaei, S. Ziaei-Rad, M. Ghayour, Vibration analysis of beams with open and breathing cracks subjected to moving masses, Journal of Sound and Vibration 326 (2009) 709-724.

[21] J. Fernández-Sáez, L. Rubio, C. Navarro, Approximate calculation of the fundamental frequency for bending vibrations of cracked beams, Journal of Sound and Vibration 225 (1999) 345-352.

[22] A. M. Ibrahim, H. Ozturk, M. Sabuncu, Vibration analysis of cracked frame structures, Structural Engineering and Mechanics 45 (2013) 3352.

[23] T. G. Chondros, A. D. Dimarogonas, Vibration of a cracked cantilever beam, Journal of vibration and acoustics 120 (1998) 742-746.

[24] M. Afshari, D. J. Inman, Continuous crack modeling in piezoelectrically driven vibrations of an Euler-Bernoulli beam, Journal of Vibration and Control 19 (2012) 341-355. 
[25] A. D. Dimarogonas, Vibration of cracked structures: A state of the art review, Engineering Fracture Mechanics 55 (1996) 831-857.

[26] M. A. C. F. Lima, Rotating Cantilever Beams : Finite Element Modeling and Vibration Analysis, Ph.D. thesis, Faculdade de Engenharia da Universidade do Porto, 2012.

[27] U. Eroglu, E. Tufekci, Exact solution based finite element formulation of cracked beams for crack detection, International Journal of Solids and Structures 96 (2016) 240-253.

[28] H. Tada, P. C. Paris, G. R. Irwin, The Stress Analysis of Cracks Handbook, Third Edition, ASME Press, New York, 2000.

[29] A. Yashar, M. Ghandchi-Tehrani, N. S. Ferguson, Dynamic behaviour of a rotating cracked beam, Journal of Physics: Conference Series 744 (2016) 012057. 